\title{
CONES IN COMPLEX AFFINE SPACE ARE TOPOLOGICALLY SINGULAR
}

DAVID PRILL

I. Introduction. A point of a complex analytic variety will be called a topological regular point (TRP), resp. an analytical regular point (ARP), if it has an open neighborhood which is homeomorphic, resp. biholomorphic, to an open set in a finite-dimensional complex vector space.

This paper shows one type of TRP is an ARP. A subvariety of $\mathbf{C}^{n}$, the $n$-dimensional complex vector space, $0<n<\infty$, is called a cone if it is a union of one-dimensional linear subspaces of $\boldsymbol{C}^{n}$.

Let $\mathbf{0}$ be the zero vector in $\mathbf{C}^{n}$. We shall prove the following:

THEOREM. If 0 is a TRP of a cone, then the cone is a linear subspace. Thus, for cones: If 0 is a TRP, then it is an $A R P$.

The idea of the proof is as follows: For $X \subset C^{n}$, let $X^{*}=X-\{0\}$. The map $p:\left(\boldsymbol{C}^{n}\right)^{*} \rightarrow \boldsymbol{C P ^ { n - 1 }}$ onto $(n-1)$-dimensional complex projective space is the projection map of a principal $C^{*}$-bundle. If $V$ is a cone, $V^{*}$ is a subbundle of $\left(\boldsymbol{C}^{n}\right)^{*}$. Propositions 1 and 2 derive topological properties of the subbundle $V^{*}$ from the assumption that 0 is a TRP of $V$. These properties guarantee $p\left(V^{*}\right)$ is a projective variety of order one. It is classical that if $p\left(V^{*}\right)$ is irreducible and of order one, then $V$ is a linear space. The lemma preceding the proof of the theorem enables us to avoid any assumptions of irreducibility.

In contrast to our result, 0 is a TRP and not an ARP for the $n$ dimensional variety

$$
\left\{\left(x_{1}, \cdots, x_{n+1}\right) \in C^{n+1} \mid x_{1}^{2}=x_{2}^{3}\right\} .
$$

A normal variety is one for which the following "removable singularities theorem" holds:

If a function is

1. defined on the points of an open set which are ARP's

2. holomorphic

3. bounded, then it can be extended to a holomorphic function on the entire open set. It is not difficult to see that a point of a one-dimensional normal variety is an ARP. Mumford [6], (see Hirzebruch [4]), has shown a TRP of a two-dimensional normal variety is an ARP. Brieskorn [1]

Received by the editors February 12, 1966. 
has observed that 0 is a TRP and not an ARP of the three-dimensional normal variety

$$
\left\{\left(x_{1}, x_{2}, x_{3}, x_{4}\right) \in C^{4} \mid x_{1}^{2}+x_{2}^{2}+x_{3}^{2}+x_{4}^{3}=0\right\} .
$$

The result of this paper is contained in the author's 1965 Princeton thesis. The author thanks his thesis advisor Professor Robert C. Gunning and Dr. Dennis Sullivan for help.

\section{Results.}

Proposition 1. Let $V$ be a k-dimensional cone for which 0 is a TRP. Then, the jth homotopy group of $V^{*}, \pi_{j}\left(V^{*}\right)$, is zero for $0 \leqq j \leqq 2 k-2$.

Proof. $V^{*}$ is clearly arcwise connected, i.e., $\pi_{0}\left(V^{*}\right)=0$. Let $U$ be a neighborhood of 0 in $V$ homeomorphic to a closed $2 k$-dimensional ball. Choose a positive $\epsilon$ so small that $N=\{z \in V|| z \mid \leqq \epsilon\} \subset U$.

Let $r$ be the deformation retraction of $V^{*}$ along lines through 0 onto $N^{*}, a$ the inclusion of $N^{*}$ in $U^{*}, b$ the inclusion of $U^{*}$ in $V^{*}$. There are homomorphisms

$$
\pi_{j}\left(V^{*}\right) \stackrel{a_{*} \circ r_{*}}{\rightarrow} \pi_{j}\left(U^{*}\right) \stackrel{b_{*}}{\rightarrow} \pi_{j}\left(V^{*}\right)
$$

Now $b \circ a \circ r$ is homotopic to the identity of $V^{*}$, so $b_{*} \circ a_{*} \circ r_{*}$ is an isomorphism. But $\pi_{j}\left(U^{*}\right)=0,1 \leqq j \leqq 2 k-2$. Hence $\pi_{j}\left(V^{*}\right)=0,0 \leqq j$ $\leqq 2 k-2$.

For $X \subset C^{n}$, let $X^{+}=p\left(X^{*}\right)$.

Proposition 2. Let $V \subset C^{n}$ be a k-dimensional cone for which the groups $\pi_{j}\left(V^{*}\right), 0 \leqq j \leqq 2 k-2$, vanish. Let $H$ be a $k$-dimensional linear subspace of $\mathbf{C}^{n}$. There is a commutative diagram of maps

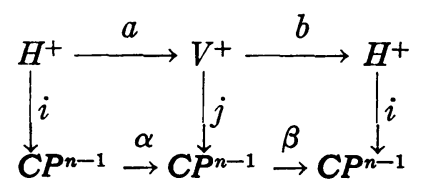

where $i$ and $j$ are inclusion maps. The composed maps $a \circ b, b \circ a$, and $\beta \circ \alpha$ are homotopic to the identity maps of their domains.

Proof. Triangulate $\mathrm{CP}^{n-1}$ so that $\mathrm{H}^{+}$and $\mathrm{V}^{+}$are finite subcomplexes [2, Satz 4, p. 199]. The hypotheses of the proposition imply that the restriction $q=p \mid V^{*}, q: V^{*} \rightarrow V^{+}$, of the bundle $p:\left(\boldsymbol{C}^{n}\right)^{*}$ $\rightarrow C P^{n-1}$ is a $(2 k-1)$-universal principal $C^{*}$-bundle [8, pp. 100-102]. Similarly, $r=p \mid H^{*}, r: H^{*} \rightarrow H^{+}$is a $(2 k-1)$-universal $C^{*}$-bundle. By the classification theorem $[8$, loc. cit. $]$ there are bundle maps $a^{*}: H^{*}$ 
$\rightarrow V^{*}, b^{*}: V^{*} \rightarrow H^{*}$. Let $a$ and $b$ be the corresponding maps of the base spaces. The bundle $p:\left(C^{n}\right)^{*} \rightarrow C P^{n-1}$ is $(2 n-1)$-universal, so $a^{*}$ and $b^{*}$ extend to maps $\alpha^{*}$ and $\beta^{*}$ of the bundle $\left(\boldsymbol{C}^{n}\right)^{*}$, which cover maps $\alpha$ and $\beta$ of $C P^{n-1}$ in to itself. The compositions $a \circ b, b \circ a, \beta \circ \alpha$ are homotopic to the identity for the same reason: They are covered by bundle maps into themselves of bundles over finite complexes which are universal for a dimension one greater than that of their base spaces. This completes the proof.

Let $H_{m}(X)$ denote the $m$ th homology group with in teger coefficients $H_{m}(X, Z)$ of the space $X$.

Corollary 1. For $0 \leqq s \leqq k-1, H_{28}\left(V^{+}\right) \cong Z$.

Proof. Proposition 2 shows $V^{+}$has the homotopy type of $H^{+}$. Thus $H_{m}\left(V^{+}\right) \cong H_{m}\left(H^{+}\right)$for all $m$. It is known [9, p. 362] that for the projective space $H^{+}, H_{2 s}\left(H^{+}\right) \cong Z$ for $0 \leqq s \leqq k-1$.

CoROllaRy 2. Let $u$ and $\nu$ be generators of $H_{2 k-2}\left(V^{+}\right)$and $H_{2 k-2}\left(H^{+}\right)$, resp. Then $j_{*}(u)= \pm i_{*}(\nu)$.

Proof. $i_{*}(\nu)=(i \circ b \circ a)_{*}(\nu)=\beta_{*}\left(j_{*}\left(a_{*}(\nu)\right)\right)$. But $a_{*}$ and $\beta_{*}$ are bijective maps and $H_{2 k-2}\left(H^{+}\right) \cong H_{2 k-2}\left(V^{+}\right) \cong H_{2 k-2}\left(C P^{n-1}\right) \cong Z$. The corollary follows.

To prove $V$ irreducible we shall use the following:

Lemma. Let $W$ be a compact complex analytic space and $X$ and $Y$ closed subvarieties with $W=X \cup Y, \operatorname{dim} X \cap Y<s$. Then, $H_{2 s}(X) \oplus H_{2 s}(Y) \cong H_{28}(W)$. The isomorphism is $i_{x} \oplus-i_{y}$, where $i_{x}$ and $i_{y}$ are the homology maps induced by the inclusions $X \subset W$, resp. $Y \subset W$.

Proof. We may triangulate $W$ so $X, Y, X \cap Y$, are subcomplexes [2, Satz 4, p. 199]. The complex $X \cap Y$ has dimension less than $2 s-1$, so

$$
H_{2 s}(X \cap Y)=H_{2 s-1}(X \cap Y)=0 .
$$

By the exact Mayer-Vietoris sequence,

$$
\begin{aligned}
0=H_{2 s}(X \cap Y) & \rightarrow H_{2 s}(X) \oplus H_{2 s}(Y) \stackrel{i_{x} \oplus-i_{y}}{\longrightarrow} H_{2 s}(W) \\
& \stackrel{\partial}{\rightarrow} H_{2 s-1}(X \cap Y)=0 .
\end{aligned}
$$

The lemma follows.

ThEOREM. Let $V \subset C^{n}$ be a cone of dimension $k$ with $\pi_{j}\left(V^{*}\right)=0$ for $0 \leqq j \leqq 2 k-2$. Then $V$ is a linear subspace of $C^{n}$. In particular when 0 
is a TRP of a cone, the cone is a linear subspace and $\mathbf{0}$ is, a fortiori, an $A R P$.

Proof. For any irreducible, compact, complex, $s$-dimensional analytic space, $H_{2 s}(X) \cong Z$ and $H_{t}(X)=0$ for all $t>2 s$ [2, Satz 8 , p. 211]. Consequently, from the lemma and Corollary 1 we conclude that exactly one branch, $V_{1}^{+}$, of $V^{+}$has dimension $(k-1)$. Moreover, the inclusion $V_{1}^{+} \subset V^{+}$induces an isomorphism of $H_{2 k-2}\left(V_{1}^{+}\right)$onto $H_{2 k-2}\left(V^{+}\right)$. Corollary 2 showed the images in $\mathrm{H}_{2 k-2}\left(\mathrm{CP}^{n-1}\right)$ of generators of $\mathrm{H}_{2 k-2}\left(V^{+}\right)$and of $\mathrm{H}_{2 k-2}\left(\mathrm{H}^{+}\right)$under the homology maps induced by the inclusions $V^{+} C \mathrm{CP}^{n-1}$ and $\mathrm{H}^{+} C \mathrm{CP}^{n-1}$ are equal (up to sign).

Select a linear subspace $L^{+}$of $C P^{n-1}$ of dimension $n-k-1$ which intersects $\mathrm{H}^{+}$transversely in one point and $V_{1}^{+}$transversely in finitely many regular points, i.e., a subspace $L^{+}$which is in general position with respect to both $H^{+}$and $V_{1}^{+}$. The topological intersection number of $L^{+}$and $V_{1}^{+}$equals (up to sign) the topological intersection number of $L^{+}$and $H^{+}, \pm 1$. (See [7, pp. 257-268].) Van der Waerden $[9$, p. 352] shows the topological and algebraic intersection numbers are equal for appropriate orientations. This means that $V_{1}^{+}$and $L^{+}$ intersect in exactly one point, i.e., $V_{1}^{+}$is of order one. It is well known [5, pp. 32-48] that if $V_{1}^{+}$is irreducible and of order one, then the corresponding cone $V_{1}$ is a linear subspace.

We now show $V_{1}=V$. We may write $V^{+}=V_{1}^{+} \cup V_{2}^{+} \cup$ $\cdots \cup V_{l}^{+} \cup E^{+}$where $V_{2}^{+}, \cdots, V_{l}^{+}$are branches of $V^{+}$of a fixed dimension $m, 0 \leqq m<k-1$, and $\operatorname{dim} E^{+}<m$. Repeated applications of the lemma show $H_{2 m}\left(V^{+}\right)$has rank $l$. By Corollary $1, l=1$. Therefore $V_{1}^{+}=V^{+}$so $V_{1}=V$ is a linear subspace.

Proposition 1 showed that if 0 is a TRP of a $k$-dimensional cone $V$, the cone satisfies $\pi_{j}\left(V^{*}\right)=0$ for $0 \leqq j \leqq 2 k-2$. By the above argument, the cone is a linear subspace and 0 is a fortiori an ARP. This completes the proof of the theorem.

\section{REFERENCES}

1. E. V. Brieskorn, Examples of singular normal complex spaces which are topological manifolds, Proc. Nat. Acad. Sci. U. S. A. 55 (1966), 1395-1397.

2. B. Giesecke, Simpliziale Zerlegungen abzählbarer analytischer Räume, Math. Z. 83 (1964), 177-213.

3. R. Gunning and H. Rossi, Analytic functions of several complex variables, Prentice-Hall, Englewood Cliffs, N. J., 1965.

4. F. Hirzebruch, The topology of normal singularities of an algebraic surface, Fasc. 250, in Seminaire Bourbaki, Vol. 15 (1962-1963), Paris, 1964.

5. W. V. D. Hodge and D. Pedoe, Methods of algebraic geometry, Vol. 2, Cambridge Univ. Press, London, 1947. 
6. D. Mumford, The topology' of normal singularities of an algebraic surface and a criterion for simplicity, Inst. Hautes Études Sci. Publ. Math. No. 9, Paris, 1961.

7. H. Seifert and W. Threlfall, Lehrbuch der Topologie, Chelsea, New York, 1947.

8. N. Steenrod, The topology of fibre bundles, Princeton Univ. Press, Princeton, N. J., 1951.

9. B. L. van der Waerden, Topologische Begründung des Kalkuls der abzählenden Geometrie, Math. Ann. 102 (1929), 337-362.

PRINCETON UNIVERSITY AND

BRANDEIS UNIVERSITY

\section{REPRESENTATION OF 0 AS $\sum_{k=-N}^{N} \epsilon_{k} k$}

J. H. VAN LINT

Abstract. If $\boldsymbol{\epsilon}_{\kappa}$ are independent identically distributed random variables with values 0 and 1 , each with probability $\frac{1}{2}$ then

$$
P\left(\sum_{k=-N}^{+N} \epsilon_{k} k=0\right) \sim\left(\frac{3}{\pi}\right)^{1 / 2} N^{-3 / 2} .
$$

1. Introduction. Recently P. Erdös asked the following question (oral communication ${ }^{1}$ ). If $A(N)$ denotes the number of representations of 0 in the form $\sum_{\boldsymbol{k}=-N}^{N} \boldsymbol{\epsilon}_{k} k$, where $\epsilon_{k}=0$ or 1 for $-N \leqq k \leqq N$ then determine the asymptotic behavior of $A(N)$. We shall prove that

$$
A(N) \sim\left(\frac{3}{\pi}\right)^{1 / 2} 2^{2 N+1} N^{-3 / 2}
$$

Another way of formulating this result is the following. Let $\epsilon_{k}$ be independent random variables identically distributed with values 0 and 1 , each with probability $\frac{1}{2}$. Then

$$
P\left(\sum_{k=-N}^{N} \epsilon_{k} k=0\right) \sim\left(\frac{3}{\pi}\right)^{1 / 2} N^{-3 / 2} .
$$

The referee has pointed out that (2) can be expected from the Lindeberg theorem as follows. If $N$ is large $\sum_{k=-N}^{N} \epsilon_{k} k$ is approximately normally distributed with mean zero and variance $N(N+1)(2 N+1) / 12$. The right hand side of (2) is the probability density at the origin of

Received by the editors June 27, 1966.

${ }^{1}$ See also [1]. 